\title{
ISOMORPHISMS OF MULTIPLIER ALGEBRAS
}

\author{
by B. J. TOMIUK
}

(Received 13 February, 1985)

1. Introduction. Let $A$ and $B$ be semisimple Banach algebras, and let $M_{l}(A)$ (resp. $M_{l}(B)$ ) be the algebra of left multipliers on $A$ (resp. $B$ ). Suppose that $A$ is an abstract Segal algebra in $B$. We find conditions on $A$ and $B$ which imply that $M_{l}(A)$ is topologically algebra isomorphic to $M_{l}(B)$. As a special case we obtain the result of [8] which states that if $A$ is an $A^{*}$-algebra that is a *-ideal in its $B^{*}$-algebra completion $B$ and $A^{2}$ is dense in $A$ then $M_{l}(A)$ is topologically algebra isomorphic to $M_{l}(B)$. We make an application of our main result to right complemented Banach algebras.

2. Preliminaries. Let $A$ be a semisimple Banach algebra. A linear mapping $T: A \rightarrow A$ is called a left multiplier if $T(x y)=T(x) y$, for all $x, y \in A$. Let $M_{l}(A)$ be the algebra of all left multipliers on $A$. Since every left multiplier on $A$ is continuous [7], $M_{l}(A)$ is a Banach algebra under the operator bound norm. For each $a \in A$, let $L_{a}$ be the operator given by $L_{a}(x)=a x$, for all $x \in A$. Then $L_{a} \in M_{l}(A)$, for all $a \in A$, and the mapping $a \rightarrow L_{A}$ is a norm-decreasing algebra isomorphism of $A$ into $M_{l}(A)$. In what follows we will identify $A$ as a subalgebra of $M_{l}(A)$.

Proposition 2.1. Let $A$ be a semisimple Banach algebra. Let $B$ be a closed subalgebra of $M_{l}(A)$ which contains $A$. Then

(i) $A$ is a left ideal of $B$,

(ii) $B$ is a semisimple Banach algebra.

Proof. (i) Let $T \in B$ and $a \in A$. Then $T(a) \in A$ and $T L_{a}(x)=T(a x)=T(a) x=$ $L_{T(a)}(x)$, for all $x \in A$. Hence, $T L_{a}=L_{T(a)}$.

(ii) Let $J$ be the radical of $B$. Since $A$ is a left ideal of $B, J \cap A$ is also a left ideal of $B$. Every $x \in J \cap A$ is left quasi-regular in $B$ and so has a left quasi-inverse in $A$. Therefore $J \cap A=(0)$ as $A$ is semisimple, so that also $J A=(0)$. Let $T \in J$. Then $0=T L_{x}=L_{T(x)}$. From the semisimplicity of $A$ we see that $T(x)=0$ for all $x$ in $A$ and so $T=0$. This completes the proof.

Let $L_{A}$ be the closure of $A$ in $M_{l}(A)$. By Proposition $2.1, L_{A}$ is a semisimple Banach algebra and contains $A$ as a dense left ideal. In the terminology of [9], $A$ is an abstract Segal algebra in $L_{A}$. We call $L_{A}$ the left regular representation of $A$.

Notation. Let $A$ and $B$ be Banach algebras such that $A$ is an abstract Segal algebra in $B$. We will denote the norm on $A(B)$ by $\|\cdot\|_{A}\left(\|\cdot\|_{B}\right)$. If $T$ is a linear map on $B$, then $T \mid A$ will denote the restriction of $T$ to $A$.

Proposition 2.2. Let $A$ be an abstract Segal algebra in a $C^{*}$-algebra $B$. Then there exists a topological algebra isomorphism of $B$ onto $L_{A}$ which maps a onto $L_{a}$, for all $a \in A$.

Proof. By [9, p. 303, Theorem 3.3], $A$ is semisimple and therefore the mapping Glasgow Math. J. 28 (1986) 73-77. 
$a \rightarrow L_{a}$ is an algebra isomorphism of $A$ into $L_{A}$. By [9, p. 299, Theorem 1.6], there exists a constant $C>0$ such that $\|b a\|_{A} \leqslant C\|b\|_{B}\|a\|_{A}$, for all $b \in B$ and $a \in A$. This shows that $\left\|L_{b}\right\| \leqslant C\|b\|_{B}$ for all $b \in A$. Hence the mapping $\varphi: a \rightarrow L_{a}$ of $A$ with the norm $\|\cdot\|_{B}$ into $L_{A}$ is continuous and extends to a continuous algebra homomorphism $\psi$ of $B$ into $L_{A}$. Let $K$ be the kernel of $\psi$. Then $K$ is a closed ideal of $B$ and $K \cap A=(0)$. Since $K A \subset K \cap A$, $K A=(0)$ and therefore $K B=(0)$. By the semisimplicity of $B, K=(0)$ and so $\psi$ is an isomorphism. By [4, p. 1104, Lemma 5.3], $\psi(B)$ is closed in $L_{A}$. Since $\psi(B)$ is dense in $L_{A}$, we obtain $\psi(B)=L_{A}$. Thus $\psi$ is onto $L_{A}$ and therefore bicontinuous. Clearly, $\psi(a)=L_{a}$, for all $a \in A$.

For a more complete treatment of $L_{A}$ see [13].

3. Main result. We first prove the following.

Proposition 3.1. Let $A$ be a semisimple Banach algebra. Then every left multiplier $S$ on $A$ has a unique extension to a left multiplier $T$ on $L_{A}$ and $\|T\| \leqslant\|S\|$.

Proof. For convenience of notation let $B=L_{A}$. Then, for every $a \in A$,

$$
\|S(a)\|_{B}=\left\|L_{S(a)}\right\|=\left\|S L_{a}\right\| \leqslant\|S\|\left\|L_{a}\right\|=\|S\|\|a\|_{B} .
$$

Therefore $S$ is bounded on $A$ with respect to the norm $\|\cdot\|_{B}$ and so has a unique extension $T$ to $B$ with $\|T\| \leqslant\|S\|$. Clearly, $T \in M_{l}(B)$ and $T \mid A=S$.

A left (right) approximate identity $\left\{u_{\alpha}\right\}$ in a Banach algebra $A$ is said to be quasi-bounded if the set $\left\{L_{u_{\alpha}}\right\}$ is bounded in $L_{A}$. It is easy to see that if $\left\{u_{\alpha}\right\}$ is a quasi-bounded left (right) approximate identity in $A$ then $\left\{L_{u_{\alpha}}\right\}$ is a bounded left (right) approximate identity in $L_{A}$.

THEOREM 3.2. Let $A$ be a semisimple Banach algebra with a quasi-bounded left approximate identity. Then $M_{l}(A)$ is topologically algebra isomorphic to $M_{l}\left(L_{A}\right)$.

Proof. For convenience of notation, let $B=L_{A}$. Let $T \in M_{l}(B)$. Since $A$ has a quasi-bounded left approximate identity, we see that $A^{2}$ is dense in $A$ and therefore, by the Hewitt-Cohen factorization theorem [6, p. 268, Theorem 32.22], $A=B \cdot A=$ $\{b a: b \in B$ and $a \in A\}$. Hence $T(A) \subseteq A$ and so $T \mid A \in M_{l}(A)\left[\mathbf{8}\right.$, p. 316]. Let $T^{\prime}=T \mid A$, and let $\left\{u_{\alpha}\right\}$ be a quasi-bounded left approximate identity in $A$. Since $T$ is continuous, there is a constant $D>0$ such that $\left\|T\left(u_{\alpha}\right)\right\|_{B} \leqslant D$ for all $\alpha$. By [9, p. 299, Proposition 1.6], there exists a constant $C>0$ such that $\|b a\|_{A} \leqslant C\|b\|_{B}\|a\|_{A}$ for all $b \in B$ and $a \in A$. Thus, for each $a \in A$,

$$
\begin{aligned}
\left\|T^{\prime}(a)\right\|_{A} & =\lim _{\alpha}\left\|T^{\prime}\left(u_{\alpha} a\right)\right\|_{A}=\lim _{\alpha}\left\|T\left(u_{\alpha}\right) a\right\|_{A} \\
& \leqslant \sup _{\alpha} C\left\|T\left(u_{\alpha}\right)\right\|_{B}\|a\|_{A} \leqslant C D\|T\|\|a\|_{A}
\end{aligned}
$$

whence $\left\|T^{\prime}\right\| \leqslant C D\|T\|$. Now, by Proposition 3.1, every $S \in M_{l}(A)$ has a unique extension $T$ to $B, T \in M_{l}(B)$ and $\|T\| \leqslant\|S\|$. Hence the mapping $T \rightarrow T^{\prime}$ is a continuous algebra isomorphism from $M_{l}\left(L_{A}\right)$ onto $M_{l}(A)$. 
Corollary 3.3. Let $A$ be an abstract Segal algebra in $B$. Assume that (i) $A^{2}$ is dense in $A$ and (ii) $B$ is semisimple and has a bounded left approximate identity contained in $A$. If $B$ is topologically algebra isomorphic to $L_{A}$ (in the sense of Proposition 2.2), then $M_{l}(A)$ is topologically algebra isomorphic to $M_{l}(B)$.

Proof. Let $\left\{u_{\alpha}\right\}$ be a bounded left approximate identity of $B$ contained in $A$. Since $A^{2}$ is dense in $A$, by [3, p. 5, Proposition 3.3], $\left\{u_{\alpha}\right\}$ is a left approximate identity of $A$. If the mapping $\psi$ of Proposition 2.2 takes $B$ onto $L_{A}$, then $\left\{u_{\alpha}\right\}$ is also a quasi-bounded left approximate identity of $A$ and $M_{l}\left(L_{A}\right)$ is topologically algebra isomorphic to $M_{l}(B)$. The conclusion now follows from Theorem 3.2.

Corollary 3.4. Let $A$ be a Banach algebra which is a dense two-sided ideal in a $B^{*}$-algebra $B$. Assume that $A^{2}$ is dense in $A$. Then $M_{l}(A)$ is topologically algebra isomorphic to $M_{l}(B)$.

Proof. By [5, p. 15, 1.7.1], $B$ has a bounded approximate identity contained in $A$. We may now apply Proposition 2.2 and Corollary 3.3 to complete the proof.

Corollary 3.5. Let $A$ be an $A^{*}$-algebra of the first kind and let $B$ be its $B^{*}$-algebra completion. Then $M_{l}(A)$ is topologically algebra isomorphic to $M_{l}(B)$.

We will now consider an application of Theorem 3.2 to right complemented Banach algebras. For the definition and basic properties of right (left) complemented Banach algebras see [11]. (See also [1], [14]).

THEOREM 3.6. Let $A$ be a semisimple annihilator right complemented Banach algebra . Then $A$ has a quasi-bounded left approximate identity.

Proof. Let $p$ denote the right complementor on $A$ and let $\left\{e_{\alpha}: \alpha \in \Omega\right\}$ be a maximal family of mutually orthogonal minimal $p$-projections in $A$. We recall that an idempotent $e$ in $A$ is called a minimal $p$-projection if $e$ is a minimal idempotent and $(e A)^{p}=(1-e) A$. Since $A$ is an annihilator algebra, every non-zero closed right ideal of $A$ contains a minimal $p$-projection. Moreover, if $e$ is a minimal $p$-projection in a closed right ideal $I$ and $f$ is a minimal $p$-projection in $I^{p}$ then $e f=f e=0$ (see [11, p. 654]). It follows that the family $\left\{e_{\alpha} A: \alpha \in \Omega\right\}$ has a dense linear span in $A$ and, for each $\alpha^{\prime} \in \Omega, e_{\alpha^{\prime}} A \cap$ $\operatorname{cl}_{A}\left(\sum_{\alpha} e_{\alpha} A\right)=(0)$. Furthermore, by $[12$, p. 268, Theorem 5.9], for every $x \in A$, $x=\sum_{\alpha \neq \alpha^{\prime}} e_{\alpha} x$, where convergence is with respect to the net of finite partial sums. Thus the family $\left\{e_{\alpha} A: \alpha \in \Omega\right\}$ forms an unconditional decomposition for $A$ and, in particular, the directed set $E$ of all finite sums $e_{\alpha_{1}}+\ldots+e_{\alpha_{n}}, \alpha_{1}, \ldots, \alpha_{n} \in \Omega$, is a left approximate identity of $A$. Therefore, by [2, p. 231, Theorem 3.4], there exists a constant $K>0$ such that, for any $\alpha_{1}, \ldots, \alpha_{n} \in \Omega$,

$$
\left\|\sum_{i=1}^{n} e_{\alpha_{i}} x\right\| \leqslant K\|x\|
$$

for all $x \in A$. Hence if we let $L_{\alpha}=L_{e_{\alpha}}, \alpha \in \Omega$, then the set of all finite sums $L_{\alpha_{1}}+\ldots+$ 
$L_{\alpha_{n}}, \alpha_{1}, \ldots, \alpha_{n} \in \Omega$, is bounded and consequently $E$ is a quasi-bounded left approximate identity of $A$.

Corollary 3.7. Let $A$ be a semisimple annihilator right complemented Banach algebra. Then $M_{l}(A)$ is topologically algebra isomorphic to $M_{l}\left(L_{A}\right)$.

Proof. This follows immediately from Theorems 3.2 and 3.6.

THEOREM 3.8. Let $A$ be a topologically simple, semisimple annihilator right complemented Banach algebra. Then there exists a Hilbert space $H$ such that $M_{l}(A)$ is topologically algebra isomorphic to $L(H)$, the algebra of all bounded linear operators on $H$.

Proof. By [1, p. 40, Theorem 1], $A$ can be continuously embedded as an abstract Segal algebra in the algebra $L C(H)$ of all compact linear operators on a Hilbert space $H$. (If $A$ is finite dimensional then $H$ is finite dimensional and the embedding is onto $L C(H)=L(H)$.) Since $L C(H)$ is a $B^{*}$-algebra, by Proposition $2.2, L_{A}$ is topologically algebra isomorphic to $L C(H)$. Therefore, by Corollary $3.7, M_{i}(A)$ is topologically algebra isomorphic to $M_{l}(L C(H))$. Observing that $M_{l}(L C(H))$ is topologically algebra isomorphic to $L(H)[10$, p. 506, Lemma 2.1] completes the proof.

Let $A$ be the algebra of trace-class operators or the algebra of Hilbert-Schmidt operators on a Hilbert space $H$. Then $A$ is a dual $A^{*}$-algebra which is a dense ${ }^{*}$-ideal in $L C(H)$. We note that $A$ is also a topologically simple, semi-simple right complemented Banach algebra. Hence $M_{l}(A)$ is topologically algebra isomorphic to $M_{l}(L C(H))$ and consequently topologically algebra isomorphic to $L(H)$.

\section{REFERENCES}

1. F. E. Alexander, On complemented and annhilator algebras, Proc. Glasgow Math. Assoc. 10 (1969), 38-45.

2. G. F. Bachelis, Homomorphisms of annihilator Banach algebras, Pacific J. Math. 25 (1968), 229-247.

3. B. A. Barnes, Banach algebras which are ideals in a Banach algebra, Pacific J. Math. 38 (1971) $1-7$.

4. S. B. Cleveland, Homomorphisms of non-commutative *-algebras, Pacific J. Math. 13 (1963), 1097-1109.

5. J. Dixmier, Les $C^{*}$-algèbres et leurs représentations (Gauthier-Villers, 1969).

6. E. Hewitt and K. A. Ross, Abstract harmonic analysis II (Springer-Verlag, 1970).

7. B. E. Johnson and A. M. Sinclair, Continuity of derivations and a problem of Kaplansky, Amer. J. Math. 90 (1968), 1067-1073.

8. D. L. Johnson and C. D. Lahr, Multipliers of $A^{*}$-algebras, Proc. Amer. Math. Soc. 74 (1979), 311-314.

9. M. Leinert, A contribution to Segal algebras, Manuscripta Math 10 (1973), 297-306.

10. B. D. Malviya and B. J. Tomiuk, Multiplier operators on $B^{*}$-algebras, Proc. Amer. Math. Soc. 31 (1972), 505-510.

11. B. J. Tomiuk, Structure theory of complemented Banach algebras, Canad. J. Math. 14 (1962), 651-659. 
12. B. J. Tomiuk and B. Yood, Topological algebras with dense socle, J. Funct. Anal. 28 (1978), 254-277.

13. B. J. Tomiuk and B. Yood, Incomplete normed algebras norms on Banach algebras, submitted.

14. B. Yood, One-sided ideals in Banach algebras, J. Nigerian Math. Soc., 1 (1982), 25-30.

Department of Mathematics

UNIVERSITY OF OTTAWA

OTTAWA

ONTARIO K1N 9B4

Canada 